\title{
A NOTE ON SUGIHARA ALGEBRAS
}

\author{
Josep M. FonT* and Gonzalo Rodríguez PÉREz*
}

\begin{abstract}
In [4] Blok and Pigozzi prove syntactically that RM, the propo sitional calculus also called R-Mingle, is algebraizable, and as a consequence there is a unique quasivariety (the so-called equivalent quasivariety semantics) associated to it. In [3] it is stated that this quasivariety is the variety of Sugihara algebras. Starting from this fact, in this paper we present an equational base for this variety abtained as a subvariety of the variety of $\mathrm{R}$-algebras, found in [7] to be associated in the same sense to the calculus $\mathbf{R}$ of relevance logic, and we determine the totally ordered, the subdirectly irreducible, and the simple members of this variety, by using some consequences of the algebraizability of the logic RM (R-Mingle) with which they are associated.
\end{abstract}

In this paper we are going to use several results found in [7] concerning the algebraizability of $\mathbf{R}$, and one of the general characterizations of algebraizability given in [4], to prove some purely algebraic properties of the variety of Sugihara algebras, the equivalent quasivariety semantics of RM. These two papers, together with the general reference [1] on Relevance Logic, form the prerequisites for the present paper.

If we start from a presentation of Relevance Logic with primitive connectives $\wedge, \rightarrow, \neg$, then the system $R M$ can be defined as the axiomatic extension of $\mathbf{R}$ with the so-called "mingle axiom"

$$
\varphi \rightarrow(\varphi \rightarrow \varphi)
$$

(see $[1$, p. 341]). We will always take by definition $\varphi \vee \psi=\neg(\neg \varphi \wedge \neg \psi)$ and $\varphi \leftrightarrow \psi=(\varphi \rightarrow \psi) \wedge(\psi \rightarrow \varphi)$. The consequence relation obtained by taking Modus Ponens $\{p, p \rightarrow q\} \vdash q$ and Adjunction $\{p, q\} \vdash p \wedge q$ as inference rules is denoted by $\vdash_{R M}$. In [4, Theorem 5.8] it is proved that both $\mathbf{R}$ and $\mathbf{R M}$ are algebraizable with the equivalence formulas

- Supported by grant PB90-0465-C02-01 of Spanish DGICYT. 
$\{p \rightarrow q, q \rightarrow p\}$ and the single defining equation $p \wedge(p \rightarrow p) \approx p \rightarrow p$. In $[7$, Theorem 17] it is proved that the equivalent quasivariety semantics for $\mathbf{R}$ is the following:

1. Definition. An algebra $\mathrm{A}=\langle A, \wedge, \rightarrow, \neg\rangle$ of type $(2,2,1)$ is called an R-algebra if and only if the following conditions are satisfied:

1. The reduct $\langle A, \wedge, \neg\rangle$ is a De Morgan lattice, with ordering $\leq$.

2. $x \rightarrow(y \rightarrow z) \leq y \rightarrow(x \rightarrow z)$ for all $x, y, z \in A$.

3. $x \leq((x \rightarrow y) \wedge z) \rightarrow y$ for all $x, y, z \in A$.

4. $x \rightarrow \neg y \leq y \rightarrow \neg x$ for all $x, y \in A$.

5. $x \rightarrow \neg x \leq \neg x$ for all $x \in A$.

6. For any $x, y, z \in A,((x \rightarrow x) \wedge(y \rightarrow y)) \rightarrow z \leq z$.

We will denote by $R$ the class of all $\mathbf{R}$-algebras (which is clearly a variety).

Algebras satisfying conditions 1 to 5 above are shown in $[7$, Theorem 5] to be definitionally equivalent to De Morgan semigroups, classically related to algebraic semantics of several relevance logics [1]; we often say that $\mathbf{R}$-algebras are De Morgan semigroups satisfying 6 above; the semigroup operation is the "intensional conjunction" or "fusion" $x * y=$ $\neg(x \rightarrow \neg y)[1$, p. 344].

If we denote by $E(A)$ the filter (in the lattice sense) generated by the set $\{x \rightarrow x: x \in A\}$, then a more useful characterization of $\mathbf{R}$-algebras is found in [7, Theorem 10]: An R-algebra is a De Morgan semigroup A such that for all $x, y \in A, x \leq y \Longleftrightarrow x \rightarrow y \in E(\mathrm{~A})$. Thus in these algebras it holds that

$$
\forall x, y \in A, \quad x=y \Longleftrightarrow x \leftrightarrow y \in E(\mathrm{~A})
$$

If $\mathrm{Z}$ denotes the set of integers, then $S=\langle Z, \wedge, \rightarrow, \neg\rangle$ will denote the algebra of type $(2,2,1)$ where $\wedge$ is the infimum with respect to the usual ordering,

$$
x \rightarrow y= \begin{cases}\neg x \vee y, & \text { if } x \leq y \\ \neg x \wedge y, & \text { if } x>y\end{cases}
$$

and $\neg x=-x$. This definition comes from Sugihara's paper [8], and in fact the algebra $S$ is sometimes called "the Sugihara algebra". Then:

2. Definition. ([3]) An algebra $A=\langle A, \wedge, \rightarrow, \neg\rangle$ of type $(2,2,1)$ is called a Sugihara algebra iff it belongs to the variety $V(S)$ generated by the algebra $S$. This variety will be denoted by $\mathrm{S}$.

3. Lemma. $\mathrm{S} \subseteq \mathrm{R}$, that is, every Sugihara algebra is an $\mathbf{R}$-algebra. 
Proof: From an observation of Dunn in [1, p. 421] and its definition of De Morgan monoid it follows that $S$ is a De Morgan semigroup; and it is also easy to check that in this case $E(\mathrm{~S})=\{z \in \mathrm{Z}: \neg z \leq z\}$. Now an easy computation using (2) shows that for any $z \in \mathrm{Z}$ and any $t \in E(\mathrm{~S})$, $t \rightarrow z \leq z$. Since for any $x, y \in \mathrm{Z}$ we have that $(x \rightarrow x) \wedge(y \rightarrow y) \in E(\mathrm{~S})$, it follows that for any $x, y, z \in Z((x \rightarrow x) \wedge(y \rightarrow y)) \rightarrow z \leq z$, that is, that $S \in R$; since $R$ is a variety, we obtain that $S=V(S) \subseteq R$.

In [3] it is mentioned that the variety of Sugihara algebras is the equivalent quasivariety semantics of RM. Since no detailed proof seems to have appeared in print, we give a simple one below, which is based on Meyer's Completeness Theorem of RM with respect to the matrix $\langle\mathrm{S}, E(\mathrm{~S})\rangle$ :

4. Theorem (Blok, Dziobiak). The variety $\mathrm{S}$ is the equivalent quasivariety semantics for RM.

Proof: We know that $\mathbf{R}$ is algebraizable and that its equivalent quasivariety semantics is a variety; since $R M$ is an axiomatic extension of $\mathbf{R}$, it follows that $\mathbf{R M}$ is also algebraizable and that its equivalent quasivariety semantics is also a variety; let us call it $K$. We also know that $\{p \rightarrow q, q \rightarrow p\}$ is a set of equivalence formulas for it; then by [4, Corollary 2.9] we know that an equation $\varphi \approx \psi$ holds in $K$ if and only if $\vdash_{\mathbf{R M}} \varphi \leftrightarrow \psi$. Then by Meyer's completeness theorem ([1, theorem 4 of section 29.3.2]) this holds if and only if $\varphi^{\mathrm{S}}(\bar{a}) \leftrightarrow \psi^{\mathrm{S}}(\bar{a}) \in E(\mathrm{~S})$ for any interpretation $\bar{a}$ of the variables of $\varphi \approx \psi$ in $\mathrm{Z}$. But by Lemma 3 we can use (1) and conclude that this holds if and only if $\varphi^{\mathrm{S}}(\bar{a})=\psi^{\mathrm{S}}(\bar{a})$, that is, if and only if the equation $\varphi \approx \psi$ holds in $\mathrm{S}$. This shows that $\mathrm{K}=V(\mathrm{~S})=\mathrm{S}$.

As a consequence we see that Sugihara algebras give us the natural algebraic semantics for RM, and this is also the case if we consider them from the point of view of logical matrices:

5. Corollary. $\langle A, T\rangle$ is a reduced matrix for $\mathrm{RM}$ if and only if $\mathrm{A} \in \mathrm{S}$ and $T=E(\mathrm{~A})$.

Proof: $\langle A, T\rangle$ is a reduced matrix for $\mathbf{R M}$ iff it is a reduced matrix for $\mathbf{R}$ which is a matrix for $\mathbf{R M}$; then the result follows from the characterization of reduced matrices for $R$ contained in Theorem 18 of $[7]$ and the general result of Corollary 5.3 of [4] which tells us that, after Theorem 4 , the algebras in $S$ are the algebraic reducts of reduced matrices for RM. 
From 5 and (1) we see that reduced matrices for RM are the so-called "normal RM-matrices" of [6]; we can thus apply to them the results of this paper. The first such application will be to clarify the relationship between Sugihara algebras and the algebras also called "Sugihara algebras" by Tokarz in [8]. Provisionally we consider the following definition:

6. Definition ([9]) An algebra $\mathrm{A}=\langle A, \wedge, \rightarrow, \neg\rangle$ of type $(2,2,1)$ will be called a "Sugihara algebra in the sense of Tokarz" iff $\langle A, \wedge, \neg\rangle$ is a De Morgan lattice totally ordered under $\leq$ such that

$$
x \rightarrow y= \begin{cases}\neg x \vee y, & \text { if } x \leq y \\ \neg x \wedge y, & \text { if } x>y\end{cases}
$$

The class of all these algebras will be denoted by $\mathrm{T}$.

The motivation for this definition is clearly the definition of $S$, the generator of Sugihara algebras, since the implication operation is determined by the same condition (2). Next we see one can characterize $\$$ starting from $\mathrm{T}$ and conversely:

7. Theorem. $\mathrm{S}$ is the variety generated by $\mathrm{T}$, and moreover for any A of type (2,2,1), $\mathrm{A} \in \mathrm{T}$ iff $\mathrm{A} \in \mathrm{S}$ and is totally ordered.

Proof: Let $\mathrm{A} \in \mathrm{T}$; we first prove that $E(\mathrm{~A})=\{x \in A: \neg x \leq x\}$ : if $x \in E(\mathrm{~A})$ there are $x_{0}, \ldots, x_{n-1} \in A$ such that $x \geq \bigwedge_{i \in n} x_{i} \rightarrow x_{i}=$ $\bigwedge_{i \in \pi} \neg x_{i} \vee x_{i}$, and since $A$ is a chain, and thus a Kleene algebra [2, p. $215]$, it follows that $\neg x \leq \bigvee_{i \in n} \neg x_{i} \wedge x_{i} \leq \bigwedge_{i \in \pi_{i}} \neg x_{i} \vee x_{i} \leq x$; conversely, if $\neg x \leq x$ then by definition $6 x \rightarrow x=\neg x \vee x=x$ and thus $x \in E(\mathrm{~A})$. As a consequence, for any $x, y \in A$, if $x \leq y$ then $x \rightarrow y=\neg x \vee y \geq$ $\neg y \wedge y \geq \neg y \wedge x=\neg(x \rightarrow y)$ and therefore $x \rightarrow y \in E(A)$; on the contrary, if $x>y$ then $x \rightarrow y=\neg x \wedge y<\neg y \wedge x \leq x \vee \neg y=\neg(x \rightarrow y)$ and thus $x \rightarrow y \notin E(\mathrm{~A})$. We conclude that A satisfies $x \leq y \Longleftrightarrow x \rightarrow y \in E(\mathrm{~A})$, and so also it satisfies (1). Now a process analogous to that followed in the proof of Theorem 4, this time using Dunn's completeness theorem of RM with respect to the class of matrices $\{\langle A, E(A)\rangle: A \in T\},[\mathbf{6}$, Theorem 10], shows that an equation holds in $S$ if and only if it holds in $\mathrm{T}$; that is, $\mathrm{S}=V(\mathrm{~T})$, the variety generated by $\mathrm{T}$. From this it follows that for any $A \in T, A \in S$ and is a chain by definition. Conversely, if $A \in S$ and is a chain, it results that $E(A)$ is a prime filter, and by $[6$, Theorem 2] we conclude that $\mathrm{A} \in \mathrm{T}$.

The following example shows that not every Sugihara algebra is a chain, that is, T ( S: Let $\mathrm{A}=\langle A, \wedge, \rightarrow, \neg\rangle$ where $A=\{0, a, e, \neg a, \neg e, 1\}$ 
has the De Morgan lattice structure determined by the Hasse diagram appearing below, and the operation $\rightarrow$ given by the table below:

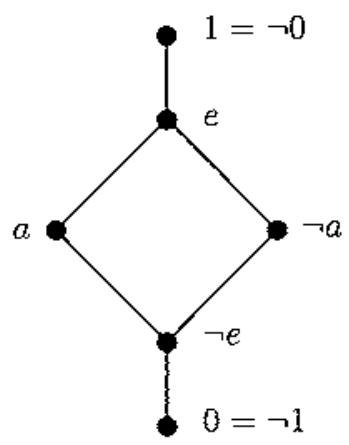

\begin{tabular}{r|rrrrrr}
$\rightarrow$ & 0 & $\neg e$ & $a$ & $\neg a$ & $e$ & 1 \\
\hline 0 & 1 & 1 & 1 & 1 & 1 & 1 \\
$\neg e$ & 0 & $e$ & $e$ & $e$ & $e$ & 1 \\
$a$ & 0 & $\neg a$ & $e$ & $\neg a$ & $e$ & 1 \\
$\neg a$ & 0 & $a$ & $a$ & $e$ & $e$ & 1 \\
$e$ & 0 & $\neg e$ & $a$ & $\neg a$ & $e$ & 1 \\
1 & 0 & 0 & 0 & 0 & 0 & 1
\end{tabular}

It is routine checking that $\Lambda \in \mathrm{S}$, and obviously it is not a chain. Note that by Theorem 7 if a Sugihara algebra is totally ordered, then its implication operation is completely determined by its De Morgan structure, namely by conditions (2); the reader should be aware, however, that in the literature (cf. $\{1$, p. 421\}), the term "Sugihara chain" has been used to denote an even more restricted class of totally ordered Sugihara algebras, namely those such that $E(A)$ has a least element $e$ (which then has to satisfy $\neg e=e$ and makes the De Morgan semigroup a monoid); again not every totally ordered Sugihara algebra satisfies this, take for instance the algebra of $\mathrm{S}$ with universe $[-1,1] \mathrm{r}\{0\}, \wedge=\min , \neg x=-x$ and $\rightarrow$ given by (2).

In [7] an equational presentation of $R$ in terms of $\wedge, \rightarrow, \neg$ is given, as an extension of one found for De Morgan semigroups; to extend it to an equational presentation of $\mathrm{S}$ we need the following technical proposition:

8. Proposition. For any $\mathrm{A} \in \mathrm{R}$ the following conditions are equivalent:

1. $x \rightarrow(x \rightarrow x) \in E(\mathrm{~A})$ for all $x \in A$;

2. $x \leq x \rightarrow x$, that is, $x \wedge(x \rightarrow x)=x$, for all $x \in A$; and

3. $E(A)=\{x \in A: x=x \rightarrow x\}=\{x \in A: \neg x \leq x\}$.

Proof: $1 \Longleftrightarrow 2:$ By $[7$, Theorem 10$)$.

$2 \Longrightarrow 3:$ By $[7$, Lemma 16] $E(A)=\{x \in A: x \rightarrow x \leq x\}$ and therefore the assumption implies $E(\mathrm{~A})=\{x \in A: x=x \rightarrow x\}$. On the other hand, if $x \in E(\mathrm{~A})$ then by assumption and [7, Proposition 4] $\neg x \leq \neg x \rightarrow \neg x=x \rightarrow x \leq x$; conversely if $\neg x \leq x$ then again by [ 7 , 
Proposition 4] $x \rightarrow x \leq \neg x \rightarrow x \leq x$ and therefore $x \in E(A)$. Thus $E(\mathrm{~A})=\{x \in A: \neg x \leq x\}$.

$3 \Longrightarrow 2$ : Consider the semigroup operation $x * y=\neg(x \rightarrow \neg y)$. Since $x \rightarrow x \in E(\mathrm{~A})$, by the assumption $x * \neg x=\neg(x \rightarrow x) \leq x \rightarrow x$ and using several computation rules of [7] we obtain $x * \neg x \leq(x * x) * \neg x=$ $x *(x * \neg x) \leq x *(x \rightarrow x) \leq x$ and then by contraposition $\neg x \leq \neg(x * \neg x)=$ $x \rightarrow x=\neg x \rightarrow \neg x$. It follows that for all $x \in A \quad x \leq x \rightarrow x$.

This result, aside from giving the equation characterizing $\mathrm{S}$, also gives us two new defining equations for the algebraizability of RM:

\section{Corollary.}

1. $\mathrm{S}$ is the subvariety of $\mathrm{R}$ determined by the equotion $p \wedge(p \rightarrow p) \approx p$.

2. RMis algebraizable with equivalence formulas $\{p \rightarrow q, q \rightarrow p\}$ and defining equation $p \approx p \rightarrow p$.

3. RMis algebraizable with equivalence formulas $\{p \rightarrow q, q \rightarrow p\}$ and defining equation $p \approx \neg p \vee p$.

4. If $\mathrm{A} \in \mathrm{S}$ and $T \subseteq A$ then $\langle\mathrm{A}, T\rangle$ is a matrix for RMif and only if $T$ is a lattice-filter of $A$ such that $T \supseteq E(A)$.

Proof: 1. Since RM is $\mathbf{R}$ plus the axiom $\varphi \rightarrow(\varphi \rightarrow \varphi)$, and using Corollary 5 and Proposition 8

2 and 3 follow from Theorem 2.4 of [4] and the completeness of RM with respect to its class of reduced matrices, given by Corollary 5

4 follows from Proposition 8 and Lemma 8 of [7].

In 2 above we note that implication alone is sufficient to write the defining equation needed for the algebraizability of RM (in contrast to the case of $\mathbf{R}$, where also conjunction is necessary); this fact was observed in [4, p. 51] for the implicational fragment of RM.

By Theorem 5.1 of [4] we know that for any algebra $A$ there is an isomorphism between the lattices $\mathcal{F}_{i} \mathrm{R}^{\mathrm{M}}$ of all filters for RM over $\mathrm{A}$, and Cons(A) of all S-congruences of A, that is, all congruences of A such that the quotient algebra belongs to $S$. This isomorphism can have interesting purely algebraic consequences, as the rest of the paper shows. Note that if $A \in S$ then $\operatorname{Con} S(A)=\operatorname{Con}(A)$ since $S$ is a variety, and $\mathcal{F}_{i} \mathrm{RM}^{\mathrm{M}}$ has been determined in the preceding Corollary as the set of all lattice filters of $\mathrm{A}$ that include $E(\mathrm{~A})$. We are going to use the following notation, for any $x \in A$ :

$$
(x, \rightarrow)=\{y \in A: x<y\} .
$$

In [3, Lemma 1.1] all finite subdirectly irreducible Sugihara algebras are determined; next we determine all subdirectly irreducible ones: 
10. Theorem. Let $\mathrm{A} \in \mathrm{S}$. Then $\mathrm{A}$ is subdirectly irreducible if and only if $\mathrm{A}$ is a chain (that is, $\mathrm{A} \in \mathrm{T}$ ) and there is a $u \in A$ such that $E(\mathrm{~A})=(u, \rightarrow)$.

Proof: $\Rightarrow$ : If $\mathrm{A} \in \mathrm{S}$ then the above-mentioned isomorphism sends the diagonal congruence to the least $\mathrm{RM}$-filter on $\mathrm{A}$, which is $E(\mathrm{~A})$ by Corollary 9,4 . 'Therefore, $A$ is subdirectly irreducible if and only if $E(\mathrm{~A})$ is completely meet-irreducible in the lattice $\mathcal{F} i \mathrm{RM}$. The characterization of RM-filters in Corollary 9 implies $E(\mathrm{~A})$ is also completely meet-irreducible as a lattice filter of $A$, and since $A$ is a distributive lattice, this implies it is a prime filter. From [6, Theorem 2] it follows that $A \in T$, that is, it is a chain. From this it follows that $E(\mathrm{~A}) \subseteq \bigcap\{(x, \rightarrow): x \in A \mathrm{r} E(\mathrm{~A})\}$. This inclusion cannot be proper, since if there is $u \in \bigcap\{(x, \rightarrow): x \in A r E(A)\}$ with $u \notin E(A)$ then $u \in(u, \rightarrow)$ which is impossible. So it must be an equality and since $E(A)$ is completely meet-irreducible in the lattice $\mathcal{F}_{i} \mathrm{RM}$ and each of these sets $(x, \rightarrow)$ belongs to it, it follows that $E(A)=(u, \rightarrow)$ for some $u \in \operatorname{Ar} E(\mathrm{~A})$, as was to be proved.

$\Longleftarrow$ : Due to the above-mentioned isomorphism, to show that $A$ is subdirectly irreducible it is enough to show that $E(\mathrm{~A})$ is completely meet-irreducible in $\mathcal{F}_{i} \AA^{\mathrm{R}}$. So suppose $E(\mathrm{~A})=\bigcap\left\{T_{i}: i \in I\right\}$ where for each $i \in I, T_{i} \in \mathcal{F}_{i}^{\mathrm{RM}}$ and $T_{i} \neq E(\mathrm{~A})$; then for each $i \in I$ there is $x_{i} \in T_{i}$ such that $x_{i} \notin E(\mathrm{~A})$, that is, $x_{i} \leq u$ since $\mathrm{A}$ is a chain by assumption. Then $u \in T_{i}$ because this is a lattice filter, and thus $u \in \bigcap\left\{T_{\imath}: i \in I\right\}=E(\mathrm{~A})=(u, \rightarrow)$ which is impossible.

Note that by the characterization of 8.3 , if $\mathrm{A} \in \mathrm{S}$ then the set $E(\mathrm{~A})$ can be called the set of all "non-negative" elements, specially when $A$ is a chain. Then we can say:

11. Corollary. Every Sugihara algebra is (isomorphic to) a subdirect product of totally ordered Sugihara algebras each hoving a greatest negative element.

Not every chain in $\mathrm{S}$ is subdirectly irreducible, as the following example shows: Take $A=[-1,1]$ the real interval, with $x \wedge y=\min \{x, y\}$ with respect the usual ordering, $\neg x=-x$, and $\rightarrow$ given by condition (2). One can check that the resulting $\mathrm{A} \in \mathrm{T}$, and that $E(\mathrm{~A})=[0,1] \neq(u, \rightarrow)=$ $(u, 1]$ for all $u \in A$. This algebra is not subdirectly irreducible, since all closed intervals $[x, 1] \in \mathcal{F}_{\mathrm{A}}^{\mathrm{RM}}$ if $x<0$, and $[0,1]=\bigcap\{[x, 1]: x<0\}$.

Wc finally determine all simple Sugihara algebras. Consider, for every $n \in \omega$, the subalgebras $S_{2 n}$ and $S_{2 n+1}$ of $S$ with universes $\{-n, \ldots,-1$, $1, \ldots, n\}$ and $\{-n, \ldots,-1,0,1, \ldots, n\}$ respectively; these are the only finite subdirectly irreducible Sugihara algebras according to [3]. Then: 
12. Theorem. The only (non-trivial) simple algebras in $\mathrm{S}$ up to isomorphism are $\mathrm{S}_{2}$ and $\mathrm{S}_{3}$, that is, the subalgebras of $\mathrm{S}$ with universes $\{-1,1\}$ and $\{-1,0,1\}$ respectively.

Proof: Since $E\left(\mathrm{~S}_{2}\right)=\{1\}$ and $E\left(\mathrm{~S}_{3}\right)=\{0,1\}$, these two algebras are obviously simple. Now let $\mathrm{A} \in \mathrm{S}$ be a simple algebra; in particular it is subdirectly irreducible, and thus it is a chain and $E(\mathrm{~A})=(u, \rightarrow)$ for some $u \in A$. On the other hand, $\operatorname{Con}(A)=\left\{\Delta_{A}, \nabla_{A}\right\}$, and thus $\mathcal{F}_{i} \mathrm{RM}=\{E(\mathrm{~A}), A\} ;$ as a consequence $u$ must be the least element of $\mathrm{A}$, and thus the only element outside $E(\mathrm{~A})$, and $\neg u$ must be the greatest. If there is another $x \in E(\mathrm{~A})$ with $x \neq \neg u$, by Proposition $8 \neg x \leq x$; if $\neg x<x=\neg \neg x$ then by the same reason $\neg x \notin E(\mathrm{~A})$, that is, $\neg x=u$ and then $x=\neg u$ which is certainly not the case; thus this $x$ must satisfy $x=\neg x$, and this can hold at most for one element in a totally ordered De Morgan lattice. Therefore $A$ is a chain with at most three elements, and thus isomorphic to one of $\mathrm{S}_{2}, \mathrm{~S}_{3}$ since implication is determined by (2).

\section{References}

1. Anderson, A. R. And Beunap, N. D.(JR.), "Entailment. The Logic of Relevance and Necessity," Princeton University Press, 1975.

2. BALBES, R. AND DWINGER, P., "Distributive lattices," University of Missouri Press, Columbia (Missouri), 1974.

3. BLOK, W. J. AND DZIOBIAK, W., On the lattice of quasivarieties of Sugihara algebras, Studia Logica 45 (1986), 275-280.

4. BLoK, W. J. AND PIGozzI, D., "Algebraizable logics," Memoirs of the American Mathematical Society 396, January 1989.

5. Burris, S. and SanKappanavar, H. P., "A Course in Univetsal Algebra," Springer-Verlag, New York, 1981.

6. DunN, J. M., Algebraic completeness results for R-minglc and its extensions, The Journal of Symbolic Logic 35 (1970), 1-13.

7. FonT, J. M. ANd Rodríguez, G., Note on algebraic models for relevance logic, Zeitschrift für Mathematische Logik und Grundlagen der Mathematik 36 (1990), 535-540.

8. Sugihara, T., Strict implication free from implicational paradoxes, Memoirs of the Faculty of Liberal Arts Fukui University, Series I (1955), 55-59. 
9. Tokarz, M., "Essays in matrix semantics of relevant logics," The Institute of Philosophy and Sociology, Polish Academy of Sciences, Warszawa, 1980.

Josep M. Font:

Departament de Lògica,

Història i Filosofia de la Ciència Facultat de Matemàtiques

Universitat de Barcelona

Gran Via 585

08007 Barcelona

SPAIN

e-mail: font@ cerber.ub.es
Gonzalo Rodríguez Pérez:

Departament de Tècniques Empresarials E. U. d'Estudis Empresarials Universitat de Barcelona

Diagonal 696

08034 Barcelona

SPAIN

Rebut el 18 de Desembre de 1991 